\title{
Chaotic Properties in the Sense of Furstenberg Families in Set-Valued Discrete Dynamical Systems
}

\author{
Xiaofang Yang1, Yongxi Jiang1, Tianxiu Lu, ${ }^{1,2^{*}}$ \\ ${ }^{1}$ College of Mathematics and Statistics, Sichuan University of Science and Engineering, Zigong, China \\ ${ }^{2}$ Key Laboratory of Higher Education of Sichuan Province for Enterprise Informationalization and Internet of Things, Zigong, \\ China \\ Email: yxf_suse@163.com, Jyx970817@163.com, ^lubeeltx@163.com
}

How to cite this paper: Yang, X.F., Jiang, Y.X. and Lu, T.X. (2021) Chaotic Properties in the Sense of Furstenberg Families in Set-Valued Discrete Dynamical Systems. Open Journal of Applied Sciences, 11, 343-353.

https://doi.org/10.4236/ojapps.2021.113025

Received: February 10, 2021

Accepted: March 27, 2021

Published: March 30, 2021

Copyright $\odot 2021$ by author(s) and Scientific Research Publishing Inc. This work is licensed under the Creative Commons Attribution International License (CC BY 4.0).

http://creativecommons.org/licenses/by/4.0/

\begin{abstract}
Let $\left(X, h_{1, \infty}\right)$ be a non-autonomous discrete system and $\left(\kappa(X), \bar{h}_{1, \infty}\right)$ be a set-valued discrete system induced by it. Where, $\kappa(X)$ is the space formed by all non-empty compact subsets of $X$ endowed with the Hausdorff metric $H, \bar{h}_{1, \infty}$ is a set-valued mapping sequence induced by $h_{1, \infty}$. It is proved that $\bar{h}_{1, \infty}$ is $\mathcal{P}$-chaos, then $h_{1, \infty}$ is $\mathcal{P}$-chaos. Where $\mathcal{P}$-chaos is denoted to $\mathcal{F}$-sensitive, $\left(\mathcal{F}_{1}, \mathcal{F}_{2}\right)$-sensitive, $\mathcal{F}$-transitive, $\mathcal{F}$-accessible, $\mathcal{F}$-weakly mixing, $\mathcal{F}-m$-sensitive, infinitely sensitive, or syndetically transitive.
\end{abstract}

\section{Keywords}

Furstenberg Family, Non-Autonomous Discrete System, $\mathcal{F}$-Sensitive, $\mathcal{F}$-Transitive, $\mathcal{F}$-Accessible, $\mathcal{F}$-Weakly Mixing, $\mathcal{F}$ - $m$-Sensitive

\section{Introduction}

As we all know, it is difficult to express some real problems using autonomous system, such as chemical problems, financial problems, biological problems, etc. Then, we need to study non-autonomous discrete systems. This research considers the following non-autonomous discrete dynamic system:

$$
x_{n+1}=h_{n}\left(x_{n}\right), n \in \mathbb{Z}^{+} .
$$

where, $h_{n}: X \rightarrow X$ is a continuous mapping sequence on compact metric space $X$ with the metric $d$. $\mathbb{Z}^{+}$is the positive integer set. Denote $h_{1, \infty}=\left\{h_{n}\right\}_{n=1}^{\infty}$. The set-valued discrete dynamic system induced system (1) is expressed as 


$$
A_{n+1}=\bar{h}_{n}\left(A_{n}\right), n \in \mathbb{Z}^{+} .
$$

where, $\bar{h}_{n}: \kappa(X) \rightarrow \kappa(X)$ is set-valued mapping sequence induced by $h_{1, \infty}$ as $h_{n}(A)=\left\{h_{n}(x) \mid x \in A\right\}$. It is obvious that $\bar{h}_{n}$ is continuous mapping sequence. $\kappa(X)$ is the space of all non-empty compact subsets of $X$ endowed with the Hausdorff metric $H$ and the Hausdorff metric on $\kappa(X)$ is defined by

$$
H(A, B)=\max \left\{\sup _{x \in A} d(x, B), \sup _{y \in B} d(y, A)\right\}
$$

For any $A, B \in \kappa(X)$.

According to [1], $(\kappa(X), H)$ is a compact metric space if and only if $(X, h)$ is a compact metric space. And by [2], for $x \in A, A \in \kappa(X)$,

$$
\begin{gathered}
B(x, \varepsilon)=\{y \in X \mid d(x, y)<\varepsilon\}, \\
B(A, \varepsilon)=\{B \in \kappa(X) \mid H(A, B)<\varepsilon\}, \\
e(A)=\{K \notin \kappa(X) \mid K \subset A\},
\end{gathered}
$$

it is clear that $e(A)=\phi$ if and only if $A=\phi$.

Through the study of the mapping sequence $h_{n}$, we understand how the points in the space $X$ move. However, it is far from enough in demographics, species migration, chemical research and numerical simulation. Sometimes, it is needed to know the movement of a finite number of point in space $X$. For example, one often iterates at the same time finite points while applying the method of numerical evaluation to the investigation of a chaotic system $\left(X, h_{1, \infty}\right)$. At this time, any set of finite points in $X$ is just an element of topological space $\kappa(X)$. Therefore, it is necessary for us to consider the set-valued mapping sequence $\bar{h}_{n}$ related to a single mapping sequence $h_{n}$. Since 2003, H. R. Flores [2] has studied the transitivity of individual mapping and set-valued mapping and many scholars have begun to study the interactions of some properties between $(X, h)$ and $(\kappa(X), \bar{h})$. A. Fedeli [3] showed that $\bar{h}$ is Devaney chaos, then $h$ is Devaney chaos. Gu and Guo [4] proved that strong mixing and mild mixing between $h$ and $\bar{h}$ are equivalent, respectively. R. Gu [5] investigated the relationships between Kato's chaoticity of the dynamical system (1) and Kato's chaoticity of the set-valued discrete system (2). J. L. G. Guirao [6] considered distributional chaos, Li-Yoeke chaos, $\omega$-chaos, Devaney chaos, topological chaos (positive topological entropy) between the dynamical system (1) and dynamical system (2). The other studies about chaotic properties of $h$ and $\bar{h}$ in autnomous systems, please refer to the literature [7] [8] [9] [10] [11]. In 2013, A. Khan [12] has investigated transitivity sensitivity and topological mixing between a non-autonomous dynamical system and its set-valued extension. In 2017, Snchez [13] studied the interactions of transitivity, weak mixing and density of periodic points between system (1) and system (2). It can be seen that there are few studies on individual chaos and set-valued chaos in non-autonomous discrete system, so it is necessary to study the chaotic relationship between $h_{1, \infty}$ and $\bar{h}_{1, \infty}$. 
The structure of this paper is as follows. In Section 2, some basic definitions are given. In Section 3, the main results are established and proved.

\section{Preliminaries}

Throughout this paper, $\left(X, h_{1, \infty}\right)$ is seen as a non-autonomous discrete dynamical system and $\left(\kappa(X), \bar{h}_{1, \infty}\right)$ is a set-valued discrete system induced by it. $\left\{h_{n}\right\}_{n=1}^{\infty}$ is a continuous self-map over the metric space $(X, d)$. A set $M \subset \mathbb{N}$ ( $\mathbb{N}$ is the natural number set) is called syndetic [14] if there exists a positive integer $p$ such that such that $\{i, i+1, \cdots, i+p\} \cap M \neq \varphi$ for any $i \in \mathbb{Z}^{+}$; a subset $T$ of $\mathbb{Z}^{+}$is said to be cofinite if there is $N \in \mathbb{Z}^{+}$such that $[N,+\infty] \subset T$; a system $\left(X, h_{1, \infty}\right)$ (or maps sequence $\left\{h_{n}\right\}_{n \in \mathbb{Z}^{+}}$) is called feebly open [15] if for any nonempty open subset $V$ of $H$, $\operatorname{int}\left(h_{n}(V)\right) \neq \phi$ for any $n \in \mathbb{Z}^{+}$; for convenience, write

$$
G(A, m, n)=\min \left\{d\left(h_{1}^{n}\left(x_{i}\right), h_{1}^{n}\left(y_{i}\right)\right): x_{i}, y_{i} \in A, i, j \in\{1,2, \cdots, m\}, i \neq j\right\}
$$

and

$$
\begin{aligned}
S_{h_{1, \infty}, m}(A, \lambda)= & \left\{n \in \mathbb{Z}^{+}, \text {there is } x_{i}, y_{i} \in A(i, j \in\{1,2, \cdots, m\}, i \neq j)\right. \\
& \text { such that } G(A, m, n) \geq \lambda\}
\end{aligned}
$$

where, $m, n \in \mathbb{Z}^{+}, A$ is any nonempty open subset in $X$.

For any $\delta>0$ and any non-empty open subset $A, B \subset X$, define

$$
\begin{gathered}
N_{h_{1, \infty}}(A, \delta)=\left\{n \in \mathbb{N}: \text { there is } x, y \in A \text { with } d\left(h_{1}^{n}(x), h_{1}^{n}(y)\right)>\delta\right\} \\
N_{h_{1, \infty}}(A, B)=\left\{n \in \mathbb{N}: h_{1}^{n}(A) \cap B \neq \varphi\right\}
\end{gathered}
$$

Definition 2.1 [16] Given an integer $m$ with $m \geq 2$. The system $\left(X, h_{1, \infty}\right)$ is called syndetic $m$ sensitive, if there is a real number $\lambda>0$ such that for any nonempty open subset $A$ of $X$, there are $2 m$ points $x_{1}, x_{2}, \cdots, x_{m} ; y_{1}, y_{2}, \cdots, y_{m} \in A$ and $n \in \mathbb{Z}^{+}$such that $S_{h_{1, \infty}, m}(A, \lambda)$ is a syndetic set.

Definition 2.2 Let $\left(X, h_{1, \infty}\right)$ be a given non-autonomous dynamical system and $\mathcal{F}$ a given Furstenberg family.

1) The system $\left(X, h_{1, \infty}\right)$ is $\mathcal{F}$-sensitive [17] with the sensitivity constant $\lambda$ if for any nonempty open subset $A$ of $X, N_{h_{1, \infty}}(A, \delta) \in \mathcal{F}$;

2) The system $\left(X, h_{1, \infty}\right)$ is $\mathcal{F}$-transitive if for any nonempty open subsets $A, B$ of $X, N_{h_{1, \infty}}(A, B) \in \mathcal{F}$;

3) The system $\left(X, h_{1, \infty}\right)$ is $\left(\mathcal{F}_{1}, \mathcal{F}_{2}\right)$-sensitive [17] with the sensitive constant $\lambda$ if for any $x \in X$ and any $\varepsilon>0$, there exist $y \in X$ with $d(x, y)<\varepsilon$ such that

$$
\left\{k \in \mathbb{Z}^{+}: d\left(h_{1}^{k}(x), h_{1}^{k}(y)\right)>\delta\right\} \in \mathcal{F}_{2}
$$

and

$$
\left\{k \in \mathbb{Z}^{+}: d\left(h_{1}^{k}(x), h_{1}^{k}(y)\right)<\delta\right\} \in \mathcal{F}_{1}
$$

for any $\delta>0$; 
4) The system $\left(X, h_{1, \infty}\right)$ is $\mathcal{F}$-accessible if for any $\varepsilon>0$ and any two nonempty open subsets $A, B \subset X$, there are two points $x \in A$ and $y \in B$ such that

$$
\left\{k: d\left(h_{1}^{k}(x), h_{1}^{k}(y)\right)<\varepsilon\right\} \in \mathcal{F} ;
$$

5) The system $\left(X, h_{1, \infty}\right)$ is $\mathcal{F}$-weakly mixing if for all nonempty open subsets $A_{i}, B_{i}, i=1,2$ of $X$ such that

$$
\left\{k: h_{1}^{k}\left(A_{1}\right) \cap B_{1} \neq \varphi \text { and } h_{1}^{k}\left(A_{2}\right) \cap B_{2} \neq \varphi\right\} \in \mathcal{F}
$$

6) The system $\left(X, h_{1, \infty}\right)$ is $\mathcal{F}$-m-sensitive if there is a real number $\lambda>0$ such that for any nonempty open subset $A$ of $X$, there are $m$ points $x_{1}, x_{2}, \cdots, x_{m} ; y_{1}, y_{2}, \cdots, y_{m} \in A$ and $n \in \mathbb{N}$ such that $S_{h_{1, \infty}, m}(A, \lambda) \in \mathcal{F}$;

7) The system $\left(X, h_{1, \infty}\right)$ is syndetically transitive [15] if for any nonempty open subsets $A, B$ of $X, N_{h_{1, \infty}}(A, B)$ is syndetic;

8) The system $\left(X, h_{1, \infty}\right)$ is infinitely sensitive [18] if there exist $\eta>0$ such that for any $y \in X$ and $\varepsilon>0$, there exists $y \in B(x, \varepsilon)$ and $n \in \mathbb{N}$ such that

$$
\limsup _{n \rightarrow \infty} d\left(h^{n}(x), h^{n}(y)\right) \geq \eta \text {. }
$$

\section{Main Result}

This section will show the relationship between $h_{1, \infty}$ and $\bar{h}_{1, \infty}$ about $\mathcal{F}$ -sensitivity, $\mathcal{F}$-sensitivity, $\mathcal{F}$-transitivity, $\mathcal{F}$-accessible, $\mathcal{F}$-weakly mixing, $\mathcal{F}$ - $m$-sensitivity, infinitely sensitivity and syndetically transitivity.

Lemma 3.1 [2] Let $A$ and $B$ be subset of $X$, then

1 ) if $A$ is a nonempty open subset of $X$, then $e(A)$ is a nonempty open subset of $\kappa(X)$;

2) $e(A \cap B)=e(A) \cap e(B)$;

3) $\bar{h}(e(A)) \subset e(h(A))$;

4) $\bar{h}^{n}=h^{n}$.

Theorem 3.2 Let $(X, d)$ be a compact metric space and $h_{1, \infty}$ be continuous self-mapping sequence on $X . \bar{h}_{1, \infty}$ is $\mathcal{F}$-sensitive, then $h_{1, \infty}$ is $\mathcal{F}$-sensitive.

Proof. Let $U$ be a nonempty open subset of $X$, the $e(U)$ be a nonempty open subset of $\kappa(X)$. Since $\bar{h}_{1, \infty}$ is $\mathcal{F}$-sensitive with the sensitive constant $\lambda>0$, then $N_{\bar{h}_{1, \infty}}(e(U), \lambda) \in \mathcal{F}$. Let $k \in N_{\bar{h}_{1, \infty}}(e(U), \lambda) \in \mathcal{F}$, by the definition, there exist $K_{1}, K_{2} \in e(U)$ with $H\left(\bar{h}_{1}^{k}\left(K_{1}\right), \bar{h}_{1}^{k}\left(K_{2}\right)\right) \geq \lambda$. Now, let $x \in U$, taking $K_{1}=\{x\} \in e(U)$. Then

$$
H\left(\bar{h}_{1}^{k}(\{x\}), \bar{h}_{1}^{k}\left(K_{2}\right)\right)=H\left(\bar{h}_{1}^{k}(x), \bar{h}_{1}^{k}\left(K_{2}\right)\right) \geq \lambda .
$$

Hence,

$$
H\left(\bar{h}_{1}^{k}(x), \bar{h}_{1}^{k}\left(K_{2}\right)\right)=\sup _{y \in K_{2}} d\left(h_{1}^{k}(x), h_{1}^{k}(y)\right) \geq \lambda
$$

According to the compactness of $K_{2}$ and the continuity of $\bar{h}_{n}(n \in \mathrm{N})$, there exists $y_{0} \in K_{2}$ such that

$$
H\left(\bar{h}_{1}^{k}(\{x\}), \bar{h}_{1}^{k}\left(K_{2}\right)\right)=d\left(h_{1}^{k}(x), h_{1}^{k}\left(y_{0}\right)\right) \geq \lambda,
$$


that is for any $U \subset X$, there exist $x, y_{0} \in U$ such that $k \in N_{h_{1, \infty}}(U, \lambda) \in \mathcal{F}$ and hence $N_{\bar{h}_{1, \infty}}(e(U), \lambda) \subset N_{h_{1, \infty}}(U, \lambda) \in \mathcal{F}$.

Thus, $h_{1, \infty}$ is $\mathcal{F}$-sensitive.

This proof has been completed.

Theorem 3.3 Let $(X, d)$ be a compact metric space and $h_{1, \infty}$ be continuous self-mapping sequence on $X . \bar{h}_{1, \infty}$ is $\left(\mathcal{F}_{1}, \mathcal{F}_{2}\right)$-sensitive, then $h_{1, \infty}$ is $\left(\mathcal{F}_{1}, \mathcal{F}_{2}\right)$ -sensitive.

Proof. Since $\bar{h}_{1, \infty}$ is $\left(\mathcal{F}_{1}, \mathcal{F}_{2}\right)$-sensitive with the sensitive constant $\lambda>0$, then for any open set $U$ of $\kappa(X)$ and any $\varepsilon>0$, there exist $V \in B(U, \varepsilon)$ such that

$$
\left\{k \in \mathbb{Z}^{+}: H\left(\bar{h}_{1}^{k}(U), \bar{h}_{1}^{k}(V)\right)>\lambda\right\} \in \mathcal{F}_{2}
$$

and

$$
\left\{k \in \mathbb{Z}^{+}: H\left(\bar{h}_{1}^{k}(U), \bar{h}_{1}^{k}(V)\right)<\lambda\right\} \in \mathcal{F}_{1} .
$$

By the definition, there are two integers $m, p \in \mathbb{N}$ such that

$$
H\left(\bar{h}_{1}^{m}(U), \bar{h}_{1}^{m}(V)\right)>\lambda \text { and } H\left(\bar{h}_{1}^{p}(U), \bar{h}_{1}^{p}(V)\right)<\lambda .
$$

Now, let $x \in X$ and $\varepsilon>0$ be given, then, taking $U=\{x\} \in \kappa(X)$. We obtain that there exists $V \in B(\{x\}, \varepsilon)$ such that

$$
H\left(\bar{h}_{1}^{m}(\{x\}), \bar{h}_{1}^{m}(V)\right)=H\left(\bar{h}_{1}^{m}(x), \bar{h}_{1}^{m}(V)\right)>\lambda
$$

and

$$
H\left(\bar{h}_{1}^{p}(\{x\}), \bar{h}_{1}^{p}(V)\right)=H\left(\bar{h}_{1}^{p}(x), \bar{h}_{1}^{p}(V)\right)<\lambda .
$$

And since

$$
H\left(\bar{h}_{1}^{k}(x), \bar{h}_{1}^{k}(V)\right)=\sup _{y \in V} d\left(h_{1}^{k}(x), h_{1}^{k}(y)\right) \geq \lambda
$$

for any $k \in \mathbb{N}$. Then, according to the compactness of $V$ and the continuity of $\bar{h}_{n}\left(n \in \mathbb{Z}^{+}\right)$, there is $y_{0} \in V$ such that

$$
H\left(\bar{h}_{1}^{m}(x), \bar{h}_{1}^{m}(V)\right)=d\left(h_{1}^{m}(x), h_{1}^{m}\left(y_{0}\right)\right)>\lambda
$$

and

$$
H\left(\bar{h}_{1}^{p}(x), \bar{h}_{1}^{p}(V)\right)=d\left(h_{1}^{p}(x), h_{1}^{p}\left(y_{0}\right)\right)>\lambda
$$

that is

$$
m \in\left\{k \in \mathbb{Z}^{+}: d\left(h_{1}^{k}(x), h_{1}^{k}\left(y_{0}\right)\right)>\lambda\right\}
$$

and

$$
p \in\left\{k \in \mathbb{Z}^{+}: d\left(h_{1}^{k}(x), h_{1}^{k}\left(y_{0}\right)\right)<\lambda\right\} .
$$

Since $U \in B(\{x\}, \varepsilon)$ implies $U \subset B(x, \varepsilon)$. And consequently, $y_{0} \in B(x, \varepsilon)$. Then

$$
\left\{k \in \mathbb{Z}^{+}: H\left(\bar{h}_{1}^{k}(U), \bar{h}_{1}^{k}(V)\right)>\lambda\right\} \subset\left\{k \in \mathbb{Z}^{+}: d\left(h_{1}^{k}(x), h_{1}^{k}\left(y_{0}\right)\right)>\lambda\right\}
$$


and

$$
\left\{k \in \mathbb{Z}^{+}: H\left(\bar{h}_{1}^{k}(U), \bar{h}_{1}^{k}(V)\right)<\lambda\right\} \subset\left\{k \in \mathbb{Z}^{+}: d\left(h_{1}^{k}(x), h_{1}^{k}\left(y_{0}\right)\right)<\lambda\right\}
$$

Thus, $h_{1, \infty}$ is $\left(\mathcal{F}_{1}, \mathcal{F}_{2}\right)$-sensitive.

This proof has been completed.

Theorem 3.4 Let $(X, d)$ be a compact metric space and $h_{1, \infty}$ be continuous self-mapping sequence on $X . \bar{h}_{1, \infty}$ is $\mathcal{F}$-transitive, then $h_{1, \infty}$ is $\mathcal{F}$-transitive.

Proof. Let $U, V$ be two nonempty open subsets of $X$, due to Lemma 3.1, $e(U)$ and $e(V)$ are nonempty open subsets of $\kappa(X)$. Since $\bar{h}_{1, \infty}$ is $\mathcal{F}$ -transitive, then $N_{\bar{h}_{1, \infty}}(e(U), e(V)) \in \mathcal{F}$. By the definition, let

$$
m \in N_{\bar{h}_{1, \infty}}(e(U), e(V)) \in \mathcal{F},
$$

then

$$
\bar{h}_{1}^{m}(e(U)) \cap e(V) \neq \phi,
$$

according to Lemma 3.1,

$$
\bar{h}_{1}^{m}(e(U)) \cap e(V)=\bar{h}_{1}^{m}(e(U) \cap e(V)) \neq \phi .
$$

Further, we obtain

$$
\bar{h}_{1}^{m}(e(U)) \cap e(V) \subseteq e\left(h_{1}^{m}(U)\right) \cap e(V)=e\left(h_{1}^{m}(U) \cap V\right) \neq \phi .
$$

Hence,

$$
h_{1}^{m}(U) \cap V \neq \phi \text { i.e. } N_{h_{1, \infty}}(e(U), e(V)) \in \mathcal{F} \text {. }
$$

Thus, $h_{1, \infty}$ is $\mathcal{F}$-transitive.

This proof has been completed.

Theorem 3.5 Let $(X, d)$ be a compact metric space and $h_{1, \infty}$ be continuous self-mapping sequence on $X$. If $\bar{h}_{1, \infty}$ is $\mathcal{F}$-accessible, then $h_{1, \infty}$ is $\mathcal{F}$-accessible.

Proof. Let $U, V$ be two nonempty open subsets of $X$, due to Lemma 3.1, $e(U)$ and $e(V)$ are nonempty open subsets of $\kappa(X)$. Since $\bar{h}_{1, \infty}$ is $\mathcal{F}$ -accessible, then for any $\varepsilon>0$, there exists $K_{1} \in e(U), K_{2} \in e(V)$ such that

$$
\left\{k \in \mathbb{Z}^{+}: H\left(\bar{h}_{1}^{k}\left(K_{1}\right), \bar{h}_{1}^{k}\left(K_{2}\right)\right)<\varepsilon\right\} \in \mathcal{F},
$$

by the definition, let

$$
m \in\left\{k \in \mathbb{Z}^{+}: H\left(\bar{h}_{1}^{k}\left(K_{1}\right), \bar{h}_{1}^{k}\left(K_{2}\right)\right)<\varepsilon\right\}
$$

one has

$$
H\left(\bar{h}_{1}^{m}\left(K_{1}\right), \bar{h}_{1}^{m}\left(K_{2}\right)\right)<\varepsilon .
$$

Now, for any $x \in U$, let $K_{1}=\{x\} \in e(U)$ then

$$
H\left(\bar{h}_{1}^{m}(\{x\}), \bar{h}_{1}^{m}\left(K_{2}\right)\right)=H\left(\bar{h}_{1}^{m}(x), \bar{h}_{1}^{m}\left(K_{2}\right)\right)<\varepsilon
$$

that is

$$
H\left(\bar{h}_{1}^{m}(x), \bar{h}_{1}^{m}\left(K_{2}\right)\right)=\sup _{y \in K_{2}} d\left(h_{1}^{m}(x), h_{1}^{m}(y)\right)<\varepsilon
$$


according to the compactness of $K_{2}$ and the continuity of $\bar{h}_{n}\left(n \in \mathbb{Z}^{+}\right)$, there is $y_{0} \in K_{2}$ such that

$$
H\left(\bar{h}_{1}^{m}(x), \bar{h}_{1}^{m}\left(K_{2}\right)\right)=d\left(h_{1}^{m}(x), h_{1}^{m}\left(y_{0}\right)\right)<\varepsilon,
$$

one has

$$
m \in\left\{k \in \mathbb{Z}^{+}: d\left(h_{1}^{m}(x), h_{1}^{m}\left(y_{0}\right)\right)<\varepsilon\right\}
$$

then

$$
\left\{k \in \mathbb{Z}^{+}: H\left(\bar{h}_{1}^{k}\left(K_{1}\right), \bar{h}_{1}^{k}\left(K_{2}\right)\right)<\varepsilon\right\} \subseteq\left\{k \in \mathbb{Z}^{+}: d\left(h_{1}^{k}(x), h_{1}^{k}\left(y_{0}\right)\right)<\varepsilon\right\} \in \mathcal{F}
$$

and hence

$$
\left\{k \in \mathbb{Z}^{+}: d\left(h_{1}^{m}(x), h_{1}^{m}\left(y_{0}\right)\right)<\varepsilon\right\} \in \mathcal{F} .
$$

Thus, $h_{1, \infty}$ is $\mathcal{F}$-accessible.

This proof has been completed.

Theorem 3.6 Let $(X, d)$ be a compact metric space and $h_{1, \infty}$ be continuous self-mapping sequence on $X . \bar{h}_{1, \infty}$ is $\mathcal{F}$-weakly mixing, then $h_{1, \infty}$ is $\mathcal{F}$ -weakly mixing.

Proof. Let $U_{i}, V_{i}(i=1,2)$ be two nonempty open subsets of $X$, due to Lemma 3.1, $e\left(U_{i}\right), e\left(V_{i}\right)(i=1,2)$ are nonempty open subsets of $\kappa(X)$. Since $\bar{h}_{1, \infty}$ is $\mathcal{F}$-weakly mixing, then

$$
\left\{k \in \mathbb{Z}^{+}: \bar{h}_{1}^{k}\left(e\left(U_{1}\right)\right) \cap e\left(V_{1}\right) \neq \varphi \text { and } \bar{h}_{1}^{k}\left(e\left(U_{2}\right)\right) \cap e\left(V_{2}\right) \neq \varphi\right\} \in \mathcal{F}
$$

Taking

$$
m \in\left\{k \in \mathbb{Z}^{+}: \bar{h}_{1}^{k}\left(e\left(U_{1}\right)\right) \cap e\left(V_{1}\right) \neq \varphi \text { and } \bar{h}_{1}^{k}\left(e\left(U_{2}\right)\right) \cap e\left(V_{2}\right) \neq \varphi\right\} \in \mathcal{F}
$$

one has

$$
\bar{h}_{1}^{m}\left(e\left(U_{1}\right)\right) \cap e\left(V_{1}\right) \neq \phi \text { and } \bar{h}_{1}^{m}\left(e\left(U_{2}\right)\right) \cap e\left(V_{2}\right) \neq \phi
$$

Due to Lemma 3.1,

$$
\overline{h_{1}^{m}}\left(e\left(U_{1}\right)\right) \cap e\left(V_{1}\right) \neq \phi \text { and } \overline{h_{1}^{m}}\left(e\left(U_{2}\right)\right) \cap e\left(V_{2}\right) \neq \phi .
$$

Further, one has

$$
e\left(h_{1}^{m}\left(U_{1}\right)\right) \cap e\left(V_{1}\right)=e\left(h_{1}^{m}\left(U_{1}\right) \cap V_{1}\right) \neq \phi
$$

and

$$
e\left(h_{1}^{m}\left(U_{2}\right)\right) \cap e\left(V_{2}\right)=e\left(h_{1}^{m}\left(U_{2}\right) \cap V_{2}\right) \neq \phi
$$

by Lemma 3.1,

$$
h_{1}^{m}\left(U_{1}\right) \cap V_{1} \neq \phi \text { and } h_{1}^{m}\left(U_{2}\right) \cap V_{2} \neq \phi .
$$

That is

$$
m \in\left\{k \in \mathbb{Z}^{+}: h_{1}^{k}\left(U_{1}\right) \cap V_{1} \neq \varphi \text { and } h_{1}^{k}\left(U_{2}\right) \cap V_{2} \neq \phi\right\}
$$

Then 


$$
\begin{aligned}
& \left\{k \in \mathbb{Z}^{+}: \bar{h}_{1}^{k}\left(e\left(U_{1}\right)\right) \cap e\left(V_{1}\right) \neq \varphi \text { and } \bar{h}_{1}^{k}\left(e\left(U_{2}\right)\right) \cap e\left(V_{2}\right) \neq \phi\right\} \\
& \subseteq\left\{k \in \mathbb{Z}^{+}: h_{1}^{k}\left(U_{1}\right) \cap V_{1} \neq \varphi \text { and } h_{1}^{k}\left(U_{2}\right) \cap V_{2} \neq \varphi\right\} \in \mathcal{F}
\end{aligned}
$$

This proves that $h_{1, \infty}$ is $\mathcal{F}$-weakly mixing.

This proof has been completed.

Theorem 3.7 Let $(X, d)$ be a compact metric space and $h_{1, \infty}$ be continuous self-mapping sequence on $X . \bar{h}_{1, \infty}$ is $\mathcal{F}$-m-sensitive, then $h_{1, \infty}$ is $\mathcal{F}$ -m-sensitive.

Proof. Let $U$ be a nonempty open subsets of $X$, then $e(U)$ is nonempty open subsets of $\kappa(X)$. Since $\bar{h}_{1, \infty}$ is $\mathcal{F}$-m-sensitive, then there exist a real number $\lambda>0$ and $m$ open sets $A_{1}, A_{2}, \cdots, A_{m} ; B_{1}, B_{2}, \cdots, B_{m} \in e(U)$ such that

$$
S_{\bar{h}_{1, \infty}, k}(e(U), \lambda) \in \mathcal{F} \text {. }
$$

Taking $p \in S_{\bar{h}_{1, \infty}, k}(e(U), \lambda)$, one has

$$
H\left(\bar{h}_{1}^{p}\left(A_{i}\right), \bar{h}_{1}^{p}\left(B_{i}\right)\right) \geq \lambda
$$

for any $i, j \in\{1,2, \cdots, m\}(i \neq j)$. That is, $p \in S_{h_{1, \infty}, k}(U, \lambda)$.

Now, let $x_{1}, x_{2}, \cdots, x_{m} \in U$, taking

$$
B_{1}=\left\{x_{1}\right\}, B_{2}=\left\{x_{2}\right\}, \cdots, B_{m}=\left\{x_{m}\right\} \in e(U)
$$

Then

$$
H\left(\bar{h}_{1}^{p}\left\{x_{i}\right\}, \bar{h}_{1}^{p}\left(B_{j}\right)\right)=H\left(\bar{h}_{1}^{p}\left(x_{i}\right), \bar{h}_{1}^{p}\left(B_{j}\right)\right) \geq \lambda
$$

for any $i, j \in\{1,2, \cdots, m\}(i \neq j)$. And since

$$
H\left(\bar{h}_{1}^{p}\left(x_{i}\right), \bar{h}_{1}^{p}\left(B_{j}\right)\right)=\sup _{y_{j} \in B_{j}} d\left(h_{1}^{p}\left(x_{i}\right), h_{1}^{p}\left(y_{j}\right)\right) \geq \lambda
$$

According to the compactness of $B_{1}=\left\{x_{1}\right\}, B_{2}=\left\{x_{2}\right\}, \cdots, B_{m}=\left\{x_{m}\right\}$ and the continuity of $\bar{h}_{n}\left(n \in \mathbb{Z}^{+}\right)$, there is $y_{1} \in B_{1}, y_{2} \in B_{2}, \cdots, y_{m} \in B_{m}$ such that

$$
H\left(\bar{h}_{1}^{p}\left\{x_{i}\right\}, \bar{h}_{1}^{p}\left(B_{j}\right)\right)=d\left(\bar{h}_{1}^{p}\left(x_{i}\right), \bar{h}_{1}^{p}\left(y_{i}\right)\right) \geq \lambda,
$$

for any $i, j \in\{1,2, \cdots, m\}(i \neq j)$.

One has that $p \in S_{h_{1, \infty}, k}(U, \lambda) \in \mathcal{F}$. This proves that $h_{1, \infty}$ is $\mathcal{F}$-m-sensitive.

This proof has been completed.

Theorem 3.8 Let $(X, d)$ be a compact metric space and $h_{1, \infty}$ be continuous self-mapping sequence on $X . \bar{h}_{1, \infty}$ is infinitely sensitive, then $h_{1, \infty}$ is infinitely sensitive.

Proof. Let $U$ be a nonempty open subsets of $X$, then $e(U)$ is nonempty open subsets of $\kappa(X)$. Since $\bar{h}_{1, \infty}$ is infinitely sensitive with the sensitive constant $\lambda>0$, then there exist $K_{1}, K_{2} \in e(U)$ such that

$$
\limsup _{k \rightarrow \infty} H\left(\bar{h}_{1}^{k}\left(K_{1}\right), \bar{h}_{1}^{k}\left(K_{2}\right)\right) \geq \lambda
$$

Now, let $x \in U$, taking $K_{1}=\{x\} \in e(U)$. Then

$$
\limsup _{k \rightarrow \infty} H\left(\bar{h}_{1}^{k}(\{x\}), \bar{h}_{1}^{k}\left(K_{2}\right)\right)=\limsup _{k \rightarrow \infty} H\left(\bar{h}_{1}^{k}(x), \bar{h}_{1}^{k}\left(K_{2}\right)\right) \geq \lambda
$$


And since

$$
H\left(\bar{h}_{1}^{k}(x), \bar{h}_{1}^{k}\left(K_{2}\right)\right)=\sup _{y \in K_{2}} d\left(h_{1}^{k}(x), h_{1}^{k}(y)\right) \geq \lambda
$$

According to the compactness of $K_{2}$ and the continuity of $\bar{h}_{n}\left(n \in \mathbb{Z}^{+}\right)$, there exists $y_{0} \in K_{2}$ such that

$$
\limsup _{k \rightarrow \infty} H\left(\bar{h}_{1}^{k}(x), \bar{h}_{1}^{k}\left(K_{2}\right)\right)=\limsup _{k \rightarrow \infty} d\left(\bar{h}_{1}^{k}(x), \bar{h}_{1}^{k}\left(y_{0}\right)\right) \geq \lambda,
$$

that is for any $U \subset X$, there exist $x, y_{0} \in U$ such that

$$
\limsup _{k \rightarrow \infty} d\left(h_{1}^{k}(x), h_{1}^{k}\left(y_{0}\right)\right) \geq \lambda
$$

Thus, $h_{1, \infty}$ is infinitely sensitive.

This proof has been completed.

Theorem 3.9 Let $(X, d)$ be a compact metric space and $h_{1, \infty}$ be continuous self-mapping sequence on $X . \bar{h}_{1, \infty}$ is syndetically transitive, then $h_{1, \infty}$ is syndetically transitive.

Proof. Let $U, V$ be two nonempty open subsets of $X$, due to Lemma 3.1, $e(U)$ and $e(V)$ are nonempty open subsets of $\kappa(X)$. Since $\bar{h}_{1, \infty}$ is syndetically transitive, then

$$
N_{\bar{h}_{1, \infty}}(e(U), e(V))=\left\{n \in \mathbb{N}: \bar{h}_{1}^{n}(e(U)) \cap e(V) \neq \varphi\right\}
$$

is syndetic. For any $m \in N_{\bar{h}_{1, \infty}}(e(U), e(V))$, one has that $\bar{h}_{1}^{m}(e(U)) \cap e(V) \neq \phi$. according to Lemma 3.1,

$$
\bar{h}_{1}^{m}(e(U)) \cap e(V)=\overline{h_{1}^{m}}(e(U)) \cap e(V) \neq \phi .
$$

Further, we obtain

$$
\overline{h_{1}^{m}}(e(U)) \cap e(V) \subseteq e\left(h_{1}^{m}(U)\right) \cap e(V) \neq \phi
$$

So, $h_{1}^{m}(U) \cap V \neq \phi$, i.e. $m \in N_{h_{1, \infty}}(U, V)$.

Thus, $N_{h_{1, \infty}}(U, V)$ is syndetic, which proves that $h_{1, \infty}$ is syndetically transitive.

This proof has been completed.

\section{Conclusion}

In set-valued discrete dynamical systems, this paper studies the chaoticity in the sense of Furstenberg families. Some sufficient conditions of $\left(\mathcal{F}_{1}, \mathcal{F}_{2}\right)$-sensitive, $\mathcal{F}$-sensitive, $\mathcal{F}$-transitive, $\mathcal{F}$-accessible, $\mathcal{F}$-weakly mixing, $\mathcal{F}$ - $m$-sensitive, infinitely sensitive, or syndetically transitive are obtained. Based on the conclusions of this paper, there are some further research in set-valued discrete dynamical systems which are worthy of studying. For example, Li-Yorke chaos, Devaney chaos, positive entropy chaos, and others.

\section{Authors and Affiliations}

The first author Xiaofang Yang, the author Yongxi Jiang have one affiliation, that is, Sichuan University of Science and Engineering. The corresponding au- 
thor Tianxiu Lu has two affiliations, that is, Sichuan University of Science and Engineering and Key Laboratory of Higher Education of Sichuan Province for Enterprise Informationalization and Internet of Things.

\section{Funding}

This work was funded by the National Natural Science Foundation of China (No. 11501391), the Opening Project of Key Laboratory of Higher Education of Sichuan Province for Enterprise Informationalization and Internet of Things (No. 2020WZJ01), the Scientific Research Project of Sichuan University of Science and Engineering (No. 2020RC24), and the Graduate student Innovation Fund (Nos. y2020077, cx2020188).

\section{Acknowledgements}

There are many thanks to the experts for their valuable suggestions.

\section{Conflicts of Interest}

The authors declare no conflicts of interest regarding the publication of this paper.

\section{References}

[1] Shao, H. and Zhu, H. (2019) Chaos in Non-Autonomous Discrete Systems and Their Induced Set-Valued Systems. Chaos, 29, 117-124. https://doi.org/10.1063/1.5054867

[2] Román-Flores, H. (2002) A Note on Transitivity in Set-Valued Discrete Systems. Chaos, Solitons \& Fractals, 17, 99-104. https://doi.org/10.1016/S0960-0779(02)00406-X

[3] Fedeli, A. (2005) On Chaotic Set-Valued Discrete Dynamical Systems. Chaos, Solitons \& Fractals, 23, 1381-1384. https://doi.org/10.1016/S0960-0779(04)00394-7

[4] Gu, R. and Guo, G. (2006) On Mixing Property in Set-Valued Discrete Systems. Chaos, Solitons \& Fractals, 28, 747-754. https://doi.org/10.1016/j.chaos.2005.04.004

[5] Gu, R. (2007) Kato's Chaos in Set-Valued Discrete Systems. Chaos, Solitons \& Fractals, 31, 765-771. https://doi.org/10.1016/j.chaos.2005.10.041

[6] Guirao, J.L.G., Kwietniak, D., Lampart, M., Oprocha, P. and Peris, A. (2009) Chaos on Hyperspaces. Nonlinear Analysis, 71, 1-8. https://doi.org/10.1016/j.na.2008.10.055

[7] Peris, A. (2005) Set-Valued Discrete Chaos. Chaos, Solitons \& Fractals, 26, 19-23. https://doi.org/10.1016/j.chaos.2004.12.039

[8] Kwietniak, D. and Oprocha, P. (2007) Topological Entropy and Chaos for Maps Induced on Hyperspaces. Chaos, Solitons \& Fractals, 33, 76-86. https://doi.org/10.1016/j.chaos.2005.12.033

[9] Li, R.S. (2012) A Note on Stronger Forms of Sensitivity for Dynamical Systems. Chaos, Solitons \& Fractals, 45, 753-758. https://doi.org/10.1016/j.chaos.2012.02.003

[10] Liao, G.F., Ma, X. and Wang, L. (2007) Individual Chaos Implies Collective Chaos for Weakly Mixing Discrete Dynamical Systems. Chaos, Solitons \& Fractals, 32, 604-608. https://doi.org/10.1016/j.chaos.2005.11.002 
[11] Liu, H., Shi, E. and Liao, G.F. (2009) Sensitivity of Set-Valued Discrete Systems. Nonlinear Analysis, 71, 6122-6125. https://doi.org/10.1016/j.na.2009.06.003

[12] Ma, X., Hou, B. and Liao, G.F. (2009) Chaos in Hyperspace System. Chaos, Solitons \& Fractals, 40, 653-660. https://doi.org/10.1016/j.chaos.2007.08.009

[13] Khan, A. and Kumar, P. (2013) Chaotic Properties on Time Varying Map and its Set Valued Extension. Advances in Pure Mathematics, 3, 359-364. https://doi.org/10.4236/apm.2013.33051

[14] Sánchez, I., Sanchis, M. and Villanueva, H. (2017) Chaos in Hyperspaces of NonAutonomous Discrete Systems. Chaos, Solitons \& Fractals, 94, 68-74. https://doi.org/10.1016/j.chaos.2016.11.009

[15] Vasisht, R. and Das, R. (2018) On Stronger Forms of Sensitivity in Non-Autonomous Systems. Taiwanese Journal of Mathematics, 22, 1139-1159. https://doi.org/10.11650/tjm/180406

[16] Zhang, C. (2016) Some Studies on m-Sensitivity Dependence in Topological Dynamical Systems. M.S. Thesis, Nanchang University, Nanchang.

[17] Li, R.S., Yu, Z., Wang, H. and Liang, H. (2020) Stronger Forms of Transitivity and Sensitivity for Non-Autonomous Discrete Dynamical Systems and Furstenberg Families. Journal of Dynamical and Control Systems, 26, 109-126. https://doi.org/10.1007/s10883-019-09437-6

[18] Wu, X.X. and Zhu, P.Y. (2012) Dense Chaos and Densely Chaotic Operators. Tsukuba Journal of Mathematics, 2, 367-375.

https://doi.org/10.21099/tkbjm/1358777004 